\title{
Gehirn ohne Geist? - armes reiches Europa!
}

\author{
Peter Heusser \\ Fakultät für Gesundheit, Department für Humanmedizin, Gerhard Kienle Lehrstuhl für Medizintheorie, \\ Integrative und Anthroposophische Medizin, Universität Witten/Herdecke, Deutschland
}

Am 28. Januar 2013 stellte die EU-Vizepräsidentin Neelie Kroes die 2 Gewinner der europäischen «Flaggschiff-Initiative» vor, eines kompetitiven Förderprojekts, von dem sich die europäische Forschung, Wirtschaft und Politik das Erreichen einer Spitzenposition Europas in der Entwicklung und Vermarktung wichtiger Zukunftstechnologien erhoffen. Mit je 1 Milliarde (!) EUR sollen die beiden gigantischen Forschungs- und Entwicklungsprojekte «Graphen» und «Human Brain Project» gefördert werden [1].

Graphen ist ein Kohlenstoffmaterial mit außergewöhnlichen Eigenschaften, von dem man unter anderem eine Revolution der heutigen Computer- und Kommunikationstechnologie erwartet. Mit dem Human Brain Project wird das Ziel verfolgt, das komplette menschliche Gehirn bis auf Molekülebene detailgetreu elektronisch nachzubauen und seine Funktionen mit einem gigantischen Supercomputer völlig neuen Typs zu simulieren. Davon erhofft man sich einen enormen Erkenntnisschub für die Gehirnforschung sowie für die Entwicklung von Computern, die das menschliche Denkvermögen nachahmen und so beispielsweise für neue Formen der Robotik einsetzbar sein sollen.

Ein wahrhaft optimistisches - wenn nicht waghalsiges Fernziel, wenn man bedenkt, dass das menschliche Gehirn etwa 100 Milliarden Neuronen enthält und trotz seines relativ bescheidenen Energieverbrauchs von umgerechnet 60 Watt gewaltig viel mehr leistet «als jeder Rechner, der Tausende von Gigawatt braucht und Milliarden Dollar kostet», so Henry Markram, der Initiator und Leiter des Human Brain Project [1]. Mediziner erhoffen sich von diesem Projekt etwa die Simulation von Medikamentenwirkungen, wodurch Tierexperimente obsolet werden sollen, sowie völlig neue Einsichten in die Entstehung und Behandlung neurodegenerativer Erkrankungen wie Alzheimer und Parkinson - ganz ähnlich wie man das auch beim «Human Genome Project» getan hat, wenn auch ziemlich vergeblich.

\section{KARGER}

Fax +497614520714

Information@Karger.com

www.karger.com (c) 2013 S. Karger GmbH, Freiburg

$1661-4119 / 13 / 0202-0100 \$ 38.00 / 0$

Accessible online at:

www.karger.com/fok
Die Euphorie ist aber vorläufig noch groß, und die EUVizepräsidentin verkündet stolz, das sei «die größte Geldprämie» für die Wissenschaft in der Geschichte Europas überhaupt. Dabei geht es klar um «Europas Wettbewerbsfähigkeit», um seine Position als «Supermacht des Wissens». Aber des Wissens wovon? Gemeint ist das umfassende Wissen der physischen Funktionsweise des menschlichen Gehirns; es geht nicht einmal um die Frage, wie aus der Gehirnfunktion Geistiges entstehe, d.h. Gedanken und menschliches Verhalten, wie ein prominenter kanadischer Neurowissenschaftler kritisiert [1].

\section{Europa als Kontinent des Gehirns?}

Immerhin binden die eingesetzten Mittel für viele Jahre wesentliche Forschungskapazitäten von führenden Wissenschaftlern aus mindestens 15 EU-Mitgliedsstaaten und fast 200 Forschungsinstituten - ein sprechendes Zeichen für das Gewicht, das man dieser Thematik in Europa beimisst.

Erstaunt das? Eigentlich nicht, denn dem Gehirn wird gegenüber dem Geist seit längerer Zeit in der Wissenschaft ohnehin Priorität eingeräumt. So vertreten auch die öffentlich tonangebenden Neurowissenschaftler des deutschsprachigen Raums wie Thomas Metzinger, Gerhard Roth und Wolf Singer die dezidierte Auffassung, der menschliche Geist mit seiner Fähigkeit des Denkens sei kausal ein Produkt des Gehirns, das «Ich» eine gesellschaftliche Konvention und die Freiheit eine Illusion [2, 3] - das Gehirn also ohne Geist. Das ist nichts wesentlich anderes, als das, was schon die Begründer der reduktionistischen Weltanschauung im 19. Jahrhunderts behauptet hatten, so etwa Rudolf Virchow 1849: «Der Naturforscher kennt nur Körper und Eigenschaften von Körpern; was darüber ist, nennt er transzendent, und die Transzendenz betrachtet er als Verirrung des menschlichen Geistes» [4]. 
(Konsequenterweise hätte er freilich sagen müssen: «...des menschlichen Gehirns», was Gerhard Roth klar auch so formulieren würde).

Was hat das alles mit Komplementärmedizin zu tun? Zunächst scheinbar gar nichts, bei näherem Zusehen aber einiges! Denn die meisten komplementärmedizinischen Richtungen machen in ihrem Organismus-, Menschen-, Krankheitsund Therapieverständnis neben den physikalisch-chemischen Kräften auch immaterielle bzw. geistige oder geistartige Formen von kausal wirkenden Kräften geltend. So spricht z.B. die Ayurvedische Medizin von «Prana», die Traditionelle Chinesische Medizin (TCM) von «Chi», die Homöopathie von «Lebenskraft», und die anthroposophische Medizin von «ätherischen» Kräften. In Virchowscher Ausdrucksweise wären diese Medizinformen also zu den «Verirrungen des menschlichen Geistes» zu zählen, und so werden sie aus der Perspektive von gewissen Vertretern des naturwissenschaftlichen Reduktionismus immer noch gesehen. Man sehe sich dazu z.B. nur einmal die Wortwahl von Homöopathiekritikern in den polemischen Auseinandersetzungen der letzten Zeit um die Wirkung homöopathischer Hochpotenzen an - es erweckt den Anschein, als würden gewisse Leute fuchsteufelswild, wenn es um die «geistartige Wirkung der Substanz» im Sinne Hahnemanns oder um andere immaterielle Wirkfaktoren in der Medizin geht.

Dass mehrere große Tageszeitungen, die immerhin als Kulturträger gelten, in letzter Zeit mit fast systematischer Regelmäßigkeit solche Polemiken publiziert und sich dabei zunehmend und in aggressivem Ton sogar gegen Universitäten gewandt haben, an denen Professuren zur kritischen Prüfung jener immateriellen Wirkfaktoren der Komplementärmedizin eingerichtet worden sind [5], kann schon nachdenklich stimmen; insbesondere wenn man diese Phänomene in der $\mathrm{Zu}-$ sammenschau mit der erwähnten Ausrichtung der Neurowissenschaften und anderen verwandten Entwicklungen betrachtet.

Was ist aus der Haltung der akademischen «Mainstream»Kultur Europas gegenüber dem Geistigen geworden? Einst war Europa ein Kontinent des Geistes, auch und gerade im akademischen Bereich. Die platonische und aristotelische Philosophie bildeten zusammen mit der christlichen Weltanschauung die Basis der europäischen Universitäten, zum Teil angestoßen durch die damalige arabische Wissenschaft. Die dabei von den Scholastikern entwickelte Denkfähigkeit bildete zusammen mit der etwa seit der Renaissance stärker geübten sinnlichen Beobachtungsfähigkeit die entscheidende Grundlage der sich neu entwickelnden empirischen Naturwissenschaften. Neben diesen behielten die Geisteswissenschaften zwar immer ihren Platz, verloren aber zunehmend an Bedeutung.

Der Reduktionismus des 19. Jahrhunderts führte schließlich zum Glauben, mit der zunehmenden Aufschlüsselung der molekularbiologischen und neurophysiologischen Bedingungen von Leben und Geist seien deren Prinzipien selbst schon erklärt und könnten aus dem wissenschaftlichen Menschenbild gestrichen werden. Im 20. Jahrhundert kapitulierten auch viele Geisteswissenschaftler vor dieser scheinbar unausweichlichen Konsequenz, allen voran die der «Philosophie des Geistes». War der «Geist» zuerst durch die mittelalterliche Spaltung zwischen Glauben und Wissen und dann durch Immanuel Kants «Ding-an-sich» ins unerreichbare Jenseits versetzt worden, so ist er jetzt ganz abgeschafft, und es bleibt das abstrakte Wissen, das vom Gehirn produziert und lediglich subjektiv sein soll. Europa hat als ein Kontinent des Gehirns und des akademischen Wissens den Geist verloren.

Aber in der Bevölkerung ist das Bedürfnis nach Geist geblieben, ja neu erwacht, auch angesichts der Inhumanität, die mit einer vornehmlich an Gen, Gehirn, Geld und Gewinn orientierten Kultur und Medizin verbunden ist. So ist es nicht verwunderlich, wenn das Bedürfnis nach Spiritualität und die Suche nach Geist tragenden Formen des Menschenverständnisses und der Medizin immer stärker geworden sind. Man muss sich ferner nicht wundern, wenn das in Europa entstandene geistige Vakuum sich mit Formen von Spiritualität und Medizin füllt, die anderen, älteren, außereuropäischen, vorchristlichen und vorwissenschaftlichen Kulturen entstammen. Zum einen ist das sehr gut, da wir in einer globalisierten Welt leben und der kulturelle Austausch zum Ausgleich von Einseitigkeiten wichtig ist. Zum andern ist aber wichtig zu bemerken, dass die Entwicklung der europäischen Wissenschaft weder mit der aktuellen Naturwissenschaft noch mit der traditionellen Geisteswissenschaft abgeschlossen ist. Letztere lässt sich erweitern und vertiefen und kann die geistige Leere von innen füllen, wenn man das weiterführt, was von bedeutenden europäischen Denkern begonnen worden ist. So haben Friedrich Wilhelm Joseph Schelling, Johann Gottlieb Fichte und Georg Wilhelm Friedrich Hegel im Gegensatz zu Immanuel Kant zunächst nachgewiesen, wie im wissenschaftlichen Bewusstsein objektiver Geist denkend tatsächlich erreichbar ist. Im Anschluss daran haben Ignaz Paul Vitalis Troxler, Immanuel Hermann Fichte und insbesondere Franz Brentano sowie Rudolf Steiner begründet und gezeigt, dass das Prinzip des empirisch beobachtenden Erkennens, wie es seit Aristoteles für die Naturwissenschaft ausgebildet worden ist, in der Fortentwicklung der Wissenschaft auch auf die Phänomene des realen Seelischen und Geistigen übertragbar ist. Demzufolge könnten auch neue, bis dahin nicht bearbeitbare geistige Erfahrungsbereiche erkenntnismäßig erschlossen und für praktische Lebensgebiete wie Psychologie und Medizin fruchtbar gemacht werden. Parallel dazu und teilweise im Zusammenhang damit haben Max Scheler, Nicolai Hartmann, Helmuth Plessner, Rudolf Steiner und andere Denker unterschiedlicher Geistesrichtungen eine natur- und geisteswissenschaftliche Anthropologie begründet, die Körper, Leben, Seele und Geist als jeweils eigenständige, wenn auch im gesunden Zustand als Einheit operierende Faktoren der menschlichen Gesamtorganisation begreift [6]. Diese Erkenntnis hat z.B. in der anthroposophischen Medizin zur Begründung einer spiri- 
tuell erweiterten naturwissenschaftlichen Medizin geführt [7] die durchaus Ähnlichkeiten mit alten spirituellen Medizinformen des Orients aufweist [8] und doch neuere europäische Wurzeln hat.

Was hat das mit dem Human Brain Project zu tun? Europa wäre trotz der Milliarden an Forschungsgeldern arm, wenn es nur ein Forschungskontinent des Gehirns bliebe und nicht auch wieder ein solcher des Geistes werden würde. Dem Bedürfnis der Patienten nach einem umfassenden, das Geistige einschließenden Menschenverständnis und einer ganzheitlichen medizinischen Betreuung muss entsprochen und die wissenschaftlichen Formen dazu müssen gefunden werden. Die Bedeutung eines auf solchen Grundlagen ruhenden Medizinsystems für die Gesundheitsverfassung der Patienten bleibt selbstverständlich zu klären. Dazu werden öffentliche Gelder nötig sein. Die Komplementärmedizin wird den FlaggschiffInitiativen sicher keine Konkurrenz machen können, und öffentliche oder gar EU-Gelder werden für die Komplementärmedizin aufgrund der herrschenden Wissenschaftsgesinnung nur zögerlich bewilligt werden. Doch darf nicht vergessen werden, dass im Unterschied zu Europa in den USA pro Jahr immerhin ca. 300 Millionen USD für komplementäre Medizin zur Verfügung stehen. Eine Hauptfrage an Politik und Wissenschaft wird sein: Welche menschliche und medizinische Kultur wollen wir eigentlich in Zukunft in Europa? Davon wird es abhängen, ob Geld nur für das Gehirn, oder auch für den Geist verfügbar sein wird.

\section{Literatur}

1 Rauner M: Ein Hauch Apollo. Die Zeit 2013;6:35.

2 Roth G: Die neurobiologischen Grundlagen von Geist und Bewusstsein; in Pauen M, Roth G (eds): Neurowissenschaften und Philosophie. Eine Einführung. München, Wilhelm Fink, 2001, pp 155209

3 Singer W: Verschaltungen legen uns fest: Wir sollten aufhören, von Freiheit zu sprechen; in Geyer C (ed): Hirnforschung und Willensfreiheit. Frankfurt/M., Suhrkamp, 2004, pp 30-65.
4 Virchow R. zit. n. Schipperges: Leib und Seele. Entwicklung von einer ganzheitlichen Schau zu einem dualistisch-analytischen Konzept; in Scheffczyk L, Staudinger H (eds): Dualismus versus Dualität: Aspekte neuzeitlicher Weltbetrachtung. Freiburg i. Br., Karl Alber, 1990, p 131.

5 Schulte von Drach MC: Alternative Heilverfahren an Hochschulen - Wissenschaft in homöopathischen Dosen. Süddeutsche Zeitung. www.sueddeutsche.de/ wissen/alternative-heilverfahren-an-hochschulenwissenschaft-in-homoeopathischen-dosen-1.1511199 (Zugriff vom 27.03.2013).
6 Heusser P: Anthroposophische Medizin und Wissenschaft. Beiträge zu einer integrativen medizinischen Anthropologie. Stuttgart, Schattauer, 2011.

7 Girke M: Innere Medizin. Grundlagen und therapeutische Konzepte der Anthroposophischen Medizin. Berlin, Salumed, 2010

8 Shin L, Zhang C: Spirituality in Traditional Chinese Medicine. Pastoral Psychol 2012;61:959-974. 